\title{
Bucindolol reduced mortality and hospitalisation related to cardiovascular causes in advanced chronic heart faillure
}

\author{
The Beta-Blocker Evaluation of Survival Trial Investigators. A trial of the beta-blocker bucindolol in patients with \\ advanced chronic heart failure. N Engl J Med 2001 May 31;344:1659-67.
}

\section{QUESTION: In patients with advanced chronic heart failure, does bucindolol reduce all-cause mortality, cardiovascular mortality, and hospitalisation for chronic heart failure?}

\section{Design}

Randomised (allocation concealed*), blinded (clinicians and patients), ${ }^{*}$ placebo controlled trial with mean follow up of 2 years (Beta-Blocker Evaluation of Survival Trial [BEST]).

Sources of funding: US National Heart, Lung, and Blood Institute;

Department of Veterans

Affairs Cooperative

Studies Program;

Incara

Pharmaceuticals

(drugs).

For correspondence:

Dr E J Eichhorn

Cardiac

Catheterization

Laboratory (IIIA2)

University of Texas

Southwestern and

Dallas Veterans Affairs

Medical Center, 4500

South Lancaster,

Dallas, TX 75216 ,

USA. Fax +1214857

1474 .

\section{Setting \\ 90 clinical sites in the USA and Canada.}

\section{Patients}

2708 patients (mean age 60 y, $78 \%$ men). Inclusion criteria were New York Heart Association (NYHA) class III or IV chronic heart failure caused by primary or secondary dilated cardiomyopathy; left ventricular ejection fraction $\leqslant 35 \%$; optimal medical treatment, including angiotensin-converting enzyme inhibitors for $\geqslant 1$ month; and $\geqslant 18$ years of age. Exclusion criteria included reversible heart failure; uncorrected primary valvular disease; active myocarditis; recent myocardial infarction or revascularisation; unstable angina; heart rate $<50$ beats/minute; or serious concomitant illness.

\section{Intervention}

Patients were allocated to bucindolol, $3 \mathrm{mg}$ twice daily for 1 week, which was then titrated gradually to a maximum dose of $100 \mathrm{mg}$ twice daily $(\mathrm{n}=1354)$ or to placebo $(\mathrm{n}=1354)$.

\section{Main outcome measures}

All-cause mortality, cardiovascular mortality, and hospitalisation related to chronic heart failure.

\section{Main results}

Analysis was by intention to treat. The groups did not differ for all-cause mortality (adjusted $\mathrm{p}=0.13$ ) (table). Patients in the bucindolol group had a lower rate of cardiovascular mortality $(\mathrm{p}=0.04)$ and hospitalisation for chronic heart failure $(\mathrm{p}<0.001)$ than did patients in the placebo group (table).

\section{Conclusions}

In patients with advanced chronic heart failure, bucindolol did not reduce all-cause mortality; however, mortality from cardiovascular causes and hospitalisation for chronic heart failure were reduced.

*See glossary.

Bucindolol v placebo for advanced chronic heart failure $(C H F) \dagger$

\begin{tabular}{lllll} 
Outcomes at mean 2 years & Bucindolol & Placebo & RRR (95\% CI) & NNT (Cl) \\
All-cause mortality & $30 \%$ & $33 \%$ & $8 \%(-2$ to 18$)$ & Not significant \\
\hline Mortality from cardiovascular diseases & $25 \%$ & $29 \%$ & $12 \%(0.4$ to 22$)$ & $29(15$ to 790$)$ \\
\hline Hospitalisation for CHF & $35 \%$ & $42 \%$ & $16 \%(8$ to 24$)$ & $15(10$ to 32$)$ \\
\hline
\end{tabular}

†Abbreviations defined in glossary; RRR, NNT, and $\mathrm{Cl}$ calculated from data in article.

\section{COMMENTARY}

Do $\beta$-blockers improve morbidity and mortality in patients with heart failure? 3 large trials have shown the benefits of bisoprolol, ${ }^{1}$ carvedilol, ${ }^{2}$ and metopro$\mathrm{lol}^{3}$ on morbidity and mortality among patients with NYHA class II or III heart failure, and have had important implications for how patients with mild-tomoderate heart failure are treated. On the basis of these studies, it has now become standard practice to treat such patients with one of these $\beta$-blockers. Whether patients with severe heart failure would also benefit from $\beta$-blockade has been unanswered. Patients with severe heart failure have the highest sympathetic outflow and theoretically may benefit most from $\beta$-blockade. These patients also have the least inotropic reserve and, thus, are most susceptible to decompensation when treated with $\beta$-blockade.

These 2 studies (BEST and COPERNICUS) have provided important data that allow us to assess the benefit of $\beta$-blockers in patients with severe heart failure. What is certain is that the benefit of $\beta$-blockers is largely dependent on the type of patients who receive them. Unfortunately, assessing the value of $\beta$-blockers in patients with severe heart failure is problematic. First, measuring and comparing severity is difficult. The NYHA functional classification is a useful guide, but, as pointed out by Braunwald, ${ }^{4}$ it is subjective and inherently imprecise. An alternative way of comparing the severity of heart failure among patients in different trials is to use placebo mortality rates. Subsets of patients in whom the annual placebo mortality rate is high (eg, 20\%) are said to have very severe heart failure. This measure also has its limitations because it does not reflect only mortality caused by heart failure and it does not include any measure of severity of symptoms, frequency of hospitalisation, or quality of life.

We are left with 2 well-designed studies, only 1 of which shows a substantial benefit of $\beta$-blocker treatment on mortality in patients with severe heart failure. These differing conclusions may have resulted from study populations that were different or from differences in the pharmacological actions of bucindolol and carvedilol. The benefits of carvedilol may be related to its unique $\alpha$-adrenergic, antioxidant, or antiendothelin effects. 


\section{Carvedilol reduced mortality and hospitalisation in severe chronic heart failure}

Packer M, Coats AJ, Fowler MB, et al, for the Carvedilol Prospective Randomized Cumulative Survival Study Group. Effect of carvedilol on survival in severe chronic heart failure. N Engl J Med 2001 May 31;344:1651-8.

\section{QUESTION: In patients with severe chronic heart failure, does carvedilol, a $\beta$-blocker, reduce mortality and hospitalisation?}

\section{Design}

Randomised \{allocation concealed*\}†, blinded (patients and clinicians),* placebo controlled trial with mean follow up of 10.4 months (Carvedilol Prospective Randomized Cumulative Survival Study [COPERNICUS]).

\section{Setting}

334 centres in 21 countries.

\section{Patients}

2289 patients (mean age 63 y, 80\% men). Inclusion criteria were dyspnoea or fatigue at rest or on minimal exertion for $\geqslant 2$ months; left ventricular ejection fraction $\leqslant 25 \%$; absence of rales and ascites; minimal or no peripheral oedema; not hospitalised for intensive care or continued inpatient care; and no recent intravenous inotropic agents or vasodilators. Exclusion criteria included chronic heart failure caused by uncorrected primary valvular disease or reversible cardiomyopathy; recent coronary revascularisation, acute myocardial, or cerebral ischaemic event, or ventricular tachycardia or fibrillation; systolic blood pressure $<85$ $\mathrm{mm} \mathrm{Hg}$; heart rate 68 beats/minute; or serum creatinine level $2.8 \mathrm{mg} / \mathrm{dl}$. Follow up was $100 \%$.

\section{Intervention}

Patients were allocated to carvedilol $3.125 \mathrm{~g}$ twice daily for 2 weeks, which was then titrated to $25 \mathrm{mg}$ twice dailyif tolerated $(n=1156)$ or to placebo $(n=1133)$. Both groups received usual medications for chronic heart failure.

\section{Main outcome measures}

Mortality and combined risk for death or hospitalisation for any reason.

\section{Main results}

Analysis was by intention to treat and used KaplanMeier survival curves. The cumulative risk for death at 1 year was lower in the carvedilol group than the placebo group (adjusted $\mathrm{p}=0.001$ ) (table). The risk for combined death or hospitalisation in the carvedilol group was lower than that in the placebo group $(\mathrm{p}<0.001)$ (table).

\section{Conclusion}

Carvedilol reduced mortality and the combined risk for death or hospitalisation in patients with severe chronic heart failure.

*See glossary.

$\nmid$ Information provided by author.
Sources of funding: Roche Pharmaceuticals and GlaxoSmithKline.

For correspondence: Dr M Packer, Division of Circulatory

Physiology, Columbia University College of Physicians and Surgeons, 630 West

168th Street, New York, NY 10032, USA. Fax

+12123057439 .

Carvedilol v placebo for severe chronic heart failure $\ddagger$

\begin{tabular}{|c|c|c|c|c|}
\hline Outcomes at 1 year & Carvedilol & Placebo & $\operatorname{RRR}(95 \% \mathrm{Cl})$ & NNT (Cl) \\
\hline Risk for death & $11 \%$ & $19 \%$ & $35 \%(19$ to 48$)$ & 15 (10 to 25$)$ \\
\hline $\begin{array}{l}\text { Combined risk for death or } \\
\text { hospitalisation }\end{array}$ & $42 \%$ & $53 \%$ & $24 \%(13$ to 33$)$ & 10 (7 to 15$)$ \\
\hline
\end{tabular}

¥Abbreviations defined in glossary; NNT and Cl calculated from data in article.

\section{COMMENTARY_continued from previous page}

The positive results from the BEST study-namely, the decrease in mortality specific to cardiovascular causes and the decrease in overall mortality among non-black patients-should not be ignored. The results of previous studies and these 2 new investigations, coupled with our increasing understanding of the role of the adrenergic nervous system in heart failure, can be used to derive a rational set of recommendations. First, patients with mild or moderate heart failure should receive $\beta$-blockers. As heart failure becomes more severe in these same patients, $\beta$-blockade must strike a delicate balance so it is forceful enough to block the adverse effects of the sympathetic nervous system and yet gentle enough to maintain any positive role this system plays in survival. For this reason, patients receiving $\beta$-blockers who develop progressive heart failure must be closely monitored. When patients with mild-to-moderate chronic heart failure who are treated with other $\beta$-blockers progress to severe heart failure, switching them to carvedilol should be considered. Additional evidence is needed before more widespread use of $\beta$-blockers in patients with severe heart failure can be recommended. Because the type of $\beta$-blocker may be very important, a trial directly comparing bucindolol and carvedilol would provide valuable evidence.

1 The Cardiac Insufficiency Bisoprolol Study II (CIBIS II): a randomised trial. Lancet 1999;353:9-13.

2 Packer M, Bristow MR, Cohn JN, et al. The effect of carvedilol on morbidity and mortality in patients with chronic heart failure. U.S. Carvedilol Heart Failure Study Group. N Engl J Med 1996;334:1349-55.

3 Metoprolol CR/XL Randomised Intervention Trial in Congestive Heart Failure (MERIT-HF). Effect of metoprolol CR/XL in chronic heart failure. Lancet 1999;353:2001-7.

4 Braunwald E. Expanding indications for beta-blockers in heart failure. N Engl J Med 2001;344:1711-2. 